Original Article

\title{
PHARMACOKINETIC STUDY OF OLOPATADINE 10 MG EXTENDED RELEASE TABLET IN COMPARISON WITH OLOPATADINE 5 MG IMMEDIATE RELEASE TABLET IN INDIAN POPULATION
}

\author{
SAIYED ZEYAUL ABRAR HUSAIN ${ }^{1}$, ARSHAD KHURO0², AMIT MARWAH ${ }^{2}$, DIVYA VOHORA
}

1Department of Pharmaceutical Medicine, Faculty of Pharmacy, Jamia Hamdard, New Delhi, India 110062, ${ }^{2}$ Ranbaxy Research Laboratories, Gurgaon, India 122015, 3Department of Pharmacology, Faculty of Pharmacy, Jamia Hamdard, New Delhi - 110062

Email: zeyaulabrar@yahoo.co.in

Received: 15 Sep 2020, Revised and Accepted: 17 Nov 2020

\begin{abstract}
Objective: This study was designed to assess the pharmacokinetics of single dose of olopatadine hydrochloride 10 mg extended release (ER) tablet of Ranbaxy laboratories limited (two test formulations) with two doses of Allelock® 5 mg immediate release (IR) tablets of Kyowa Hakko Kogyo Co. Ltd. (reference formulation R), in healthy, adult, Indian male subjects under fed condition.

Methods: Fifteen healthy male volunteers, $26.07 \pm 6.62$ y in age and $57.17 \pm 6.68 \mathrm{~kg}$ in body weight, were divided into three groups and received either olopatadine hydrochloride $10 \mathrm{mg}$ ER tablet or two doses of Allelock® $5 \mathrm{mg}$ tablets in each period. Blood samples were taken at predetermined time points and plasma concentrations of olopatadine were monitored by liquid chromatography mass spectrometric (LCMS/MS). Pharmacokinetic (PK) parameters AUC0-t, AUC0-24, AUC0- $\infty$, and Cmax were calculated for olopatadine using WinNonlin. A statistical analysis was performed on PK data using SAS system.
\end{abstract}

Results: The ER formulations showed a similar AUC as compared to the IR formulation and there was no statistically significant difference in AUC of test formulation A and B and reference R. The ratios of AUC0-t, AUC0-24 and AUC0- $\infty$ for A/R were 91.08, 94.90 and 91.32 and for B/R were 89.63, 93.95 and 89.63 respectively. The ER formulations reported a higher Cmax value as compared to IR formulation. The ratios of Cmax for A/R and B/R were 151.09 and 167.96 respectively. But these higher Cmax values did not pose any safety issue as there were no serious adverse events reported during the study.

Conclusion: In conclusion, we can say that though the study drugs did not meet the bioequivalence criteria set by regulatory agencies, but this study gave an insight about PK properties of olopatadine extended release formulation and given an idea about effect of smoking on the PK profile of olopatadine which can be studied in future.

Keywords: Olopatadine, Antihistaminic, Antiallergic, Pharmacokinetic, Bioavailability, Extended-release, Smoking

(C) 2021 The Authors. Published by Innovare Academic Sciences Pvt Ltd. This is an open access article under the CC BY license (https://creativecommons.org/licenses/by/4.0/) DOI: https://dx.doi.org/10.22159/ijcpr.2021v13i1.40820. Journal homepage: https://innovareacademics.in/journals/index.php/ijcpr

\section{INTRODUCTION}

Extended release (ER) or modified release (MR) mode of drug administration has certain advantageous impact on the magnitude of the pharmacologic response: (a) it minimizes fluctuation in blood drug concentrations', (b) it produces a slow input rate which tends to minimize the body's counteraction to the drug's intervening effect on regulated physiological processes; and (c) it provides a continuous mode of drug administration [1]. ER or MR formulations provide higher maximum plasma concentrations with lower inter-patient variability than the conventional, immediate release (IR), twice-daily formulations. Additionally, therapeutic drug levels with ER formulations achieved rapidly and maintained over the course of $24 \mathrm{~h}$, allowing once-daily dosing. The studies have also confirmed good tolerability and safety of ER formulations similar to the IR formulations [2]. Another undoubted advantage of ER formulation is improved patient compliance. Compliance improves dramatically as prescribed dose frequency decreases [3-6]. The therapeutic effectiveness of a drug depends upon its bioavailability to elicit the desired pharmacological response. There are many drug-related (physicochemical properties) and host factors (physiological factors like age, blood flow to gastrointestinal tract (GIT), $\mathrm{pH}$, gastric emptying etc.) that influence the rate and absorption of the drugs [7].

Allergic rhinoconjunctivitis and chronic urticaria, as well as eczema and bronchial asthma, are associated with a hypersensitive response of the immune system. This occurs following the interaction of allergen with a specific antibody that has been adsorbed onto the surface of mast cells and basophils located in the tissue and blood, respectively. Allergic rhinitis and conjunctivitis, which are the most common forms of atopic disease, are characterized by sneezing, rhinorrhoea, nasal obstruction and itching of the nose and eyes. There are many antiallergic and antihistaminic drugs for the treatment of rhinoconjunctivitis, urticaria, eczema and bronchial asthma. However, the incidence of these allergic diseases in general has been increasing. As the prevalence of these allergic diseases rises, efforts at the discovery of novel and effective medications for prevention and treatment of these conditions also rise [8].

Olopatadine hydrochloride is a novel antiallergic/histamine H1receptor antagonist. It is a potent histamine H1-receptor antagonist and a specific mast cell stabilizer, with additional anti-inflammatory properties. Olopatadine hydrochloride principally acts as a selective histamine $\mathrm{H} 1$ receptor antagonist. Olopatadine is indicated for allergic rhinitis, urticaria, itching resulting from skin diseases (eczema/dermatitis, prurigo, pruritus cutaneous, psoriasis vulgaris, multiform exudative erythema) [9].

Olopatadine is approved as an ophthalmic solution in Europe, Japan, and the United States. In Japan, the molecule is also available as an oral formulation (Allelock®; Kyowa Hakko Kirin Co., Ltd., Tokyo, Japan) for treatment of allergic conditions9. Allelock ${ }^{\circledR}$ conventional tablets are currently administered twice daily, in the morning and before going to bed. Hence, it is advantageous to formulate once daily dosage regimen for olopatadine hydrochloride as it will exhibit better patient compliance in outpatient therapy. Therefore, the present study was designed to evaluate bioavailability of single dose of olopatadine hydrochloride 10 mg extended release tablet (two formulations) of Ranbaxy laboratories limited in comparison with two doses of Allelock $₫ 5 \mathrm{mg}$ tablets of Kyowa Hakko Kogyo Co. Ltd., in healthy, adult, human male subjects under fed condition. This is the first study of olopatadine hydrochloride $10 \mathrm{mg}$ extended release tablet in Indian population. 


\section{MATERIALS AND METHODS}

\section{Study design}

The study was carried out in accordance with the basic principles defined in US 21CFR Part 320, USFDA guidance for industry for conducting bioavailability and bioequivalence Studies for orally administered drug products--general consideration 2003, ICH (62 FR25692, 09 May 1997)' Guidance for 'Good Clinical Practice', ICMR 'ethical guidelines for biomedical research on human participants (2006)', CDSCO 'guidance for Good Clinical Practices for Clinical Research in India' and the principles enunciated in the Declaration of Helsinki [10-14]. The study protocol was approved by the Jamia Hamdard Institutional Review Board. Adequate numbers of subjects were selected randomly from the volunteer bank of the clinical pharmacology unit (CPU) and all the subjects underwent a standardized screening procedure. All subjects were informed with objectives, treatments, potential risks, dates and activities during the clinical part of study. A written consent form was signed by each enrolled subject.

The study was designed as an open label, balanced, randomized, three-treatment, three-period, three-sequence, crossover comparative bioavailability study in healthy, adult, human male subjects under fed condition. During all the three periods of the study, subjects reported to CPU at least $12 \mathrm{~h}$ before dose administration on day 1 . After sampling for $36 \mathrm{~h}$ post dose as per schedule, subjects were discharged on the evening of day 2. Postadmission to CPU during all the three periods of the study, all subjects were fasted overnight for at least $10 \mathrm{~h}$ before the high-fat high-calorie breakfast. There was $6 \mathrm{~d}$ washout period between the administrations of study drugs in each period.

\section{Study drugs}

The test product were two formulations of olopatadine hydrochloride $10 \mathrm{mg}$ extended release tablet manufactured by Ranbaxy Laboratories Limited, India (batch number RV (4430)01, RV (4430)05, expiry date February 2012) and the reference product was Allelock ${ }^{\circledR} 5$ mg tablet manufactured and distributed by Kyowa Hakko Kogyo Co. Ltd., Japan (batch number 109AIH, expiry date August 2012). Morning dose was administered with $240 \mathrm{ml}$ of drinking water at ambient temperature, 45 min after starting of high fat high calorie breakfast in all the three periods of the study. In case of reference product, evening dose was administered with $240 \mathrm{ml}$ of drinking water at ambient temperature, $45 \mathrm{~min}$ after starting of high fat high calorie dinner in all the three periods of the study. The order of receiving study treatments for each subject during the three periods of the study was determined according to the SAS-generated balanced randomization schedule.

\section{Study subjects}

Subjects who have voluntarily given written informed consent to participate in this study were selected based on inclusion/exclusion criteria like age range of 18-45 y, neither overweight nor underweight for his height and have haemoglobin $\geq 13.0 \mathrm{~g} / \mathrm{dl}$ etc. Medical history and demographic data, including name, sex, age, body weight (kg), height $(\mathrm{cm})$ and tobacco use (including number of cigarettes smoked per day) were recorded. Each subject underwent physical examination and the laboratory tests of hematologic, hepatic/renal functions, urinalysis, serology and ECG. Fifteen medically healthy subjects with clinically normal laboratory profiles were selected based on the inclusion/exclusion criteria as per protocol. The subjects did not take any prescription, OTC medications and vitamins for at least $30 \mathrm{~d}$ prior to the start of study and during the study. All the subjects consumed the meal as per the meal schedule and abstained from any alcohol/products containing alcohol and grapefruit juice and/or grapefruit supplements for $48 \mathrm{~h}$ prior to admission and till last sample collection for pharmacokinetic analysis in all the three periods of the study. Subjects also abstained from tea, coffee, cigarette and any other xanthine containing beverages, during in-house stay in all the three periods of the study.

During the study periods, all the subjects were under medical supervision. Vital signs were examined at scheduled time as per the protocol.

\section{Blood sampling}

Blood samples from each subject were collected in prechilled K3 ethylenediamine tetraacetic acid (EDTA) vacutainers through indwelling heparinized cannula placed in forearm veins.

The blood samples were collected pre-dose (in duplicate) and at $0.250,0.500,0.750,1.000,1.500,2.000,2.500,3.000,3.500,4.000$, $5.000,6.000,7.000,8.000,10.000,12.000,16.000,24.000,30.000$ and $36.000 \mathrm{~h}$ post-dose from subject of test-arm and at $0.167,0.250$, $0.333,0.500,0.667,0.833,1.000,1.333,1.667,2.000,2.500,3.000$, $4.000,6.000,8.000,10.000,12.000,12.167,12.250,12.333,12.500$, $12.667,12.833,13.000,13.333,13.667,14.000,14.500,15.000$, $16.000,18.000,20.000,24.000,30.000$ and $36.000 \mathrm{~h}$ post morning dose from subjects of reference-arm in all the three periods of the study.

The pre-dose blood samples in all the three periods of the study were collected within a period of approximately $1.5 \mathrm{~h}$ before the morning dose and the post-dose samples were generally collected within $2 \mathrm{~min}$ of the scheduled time. After collection, the blood samples were centrifuged at a speed of 4000 RPM for duration of 15 min and at a temperature of $4 \pm 2{ }^{\circ} \mathrm{C}$ under refrigeration as soon as possible to separate plasma. All post dose plasma samples were divided into two aliquots and transferred to suitably labelled tubes. The plasma samples were then stored at below $-50{ }^{\circ} \mathrm{C}$, pending transfer to the analytical facility for assay.

\section{Sample analysis}

A liquid chromatography mass spectroscopy (LC-MS/MS) method for the estimation of olopatadine in human plasma was developed and validated by using Olopatadine- $\mathrm{d} 3$ as internal standard (ISTD). The validation of this procedure was performed to evaluate the method in terms of selectivity, linearity, precision, accuracy, sensitivity, recovery and stability [15]. The procedure involved solid phase extraction with oasis HLB 1CC cartridges. The drug and the ISTD were eluted from a Zorbax eclipse XDB C18, 100x4.6 mm, $3.5 \mu \mathrm{M}$ column at $30{ }^{\circ} \mathrm{C}$ with a mobile phase consisting of $0.02 \%$ formic acid solution: methanol $(40: 60)(\mathrm{v} / \mathrm{v})$ at a flow rate of 1 $\mathrm{ml} / \mathrm{min}$. Mass spectrometric detector was used to measure the drug and ISTD using multiple reaction monitoring. Each analysis requires no longer than $2.5 \mathrm{~min}$. Quantification was achieved by measurement of the peak area ratio of the drug to the ISTD. The limit of the quantification of olopatadine in human plasma was $1.0023 \mathrm{ng} / \mathrm{ml}$.

\section{Pharmacokinetic analysis}

The concentration data obtained from analytical study was entered in WinNonlin pharmacokinetic software for further processing. The PK parameters were calculated for olopatadine using WinNonlin Node version 5.0.1 from Pharsight. Area under the curve (AUC) values like $\mathrm{AUC}_{0-\mathrm{t}}$ (AUC from time zero to the last measurable concentration), $\mathrm{AUC}_{0-24}$ (AUC from time zero to $24 \mathrm{~h}$ ) were calculated by the linear trapezoidal method. $\mathrm{AUC}_{0-\infty}$ (AUC from time zero to infinity) is calculated as the sum of $\mathrm{AUC}_{0-\mathrm{t}}$ plus the ratio of the last measurable plasma concentration to the elimination rate constant. AUC $_{\% \text { Extrap }}$ (the percentage of extrapolated AUC from the last measurable concentration to infinity) was calculated as [(AUC $0-\infty-$ $\left.\left.\mathrm{AUC}_{0-\mathrm{t}}\right) / \mathrm{AUC}_{0-\infty}\right]^{*} 100$. The maximum plasma concentration $\left(\mathrm{C}_{\max }\right)$ and the time to reach $\mathrm{C}_{\max }\left(\mathrm{T}_{\max }\right)$ were taken directly from observed concentration vs time data. Kel (elimination rate constant) was calculated from a semi-log plot of the plasma concentration versus time curve. The parameter was calculated by linear least-square regression analysis using the maximum number of points in the terminal log-linear phase (e. g. three or more non-zero plasma concentrations). $\mathrm{T}_{1 / 2}$ (elimination half-life) was calculated as $0.693 / \mathrm{K}_{\mathrm{el}}$.

\section{Statistical analysis}

Statistical analysis was performed using the WinNonlin PK Software, Version 5.0.1. The analysis included the data from all subjects who have completed the study. Arithmetic means, standard deviations and coefficients of variation were calculated for the abovementioned PK parameters. Additionally, geometric means and percentage coefficient 
of variation of geometric means was calculated for $\mathrm{AUC}_{0-\mathrm{t}}, \mathrm{AUC}_{0-24}$, $\mathrm{AUC}_{0-\infty}$ and $\mathrm{C}_{\text {max }}$. The log-transformed $\mathrm{PK}$ parameters $\left(\mathrm{C}_{\max }, \mathrm{AUC}_{0-\mathrm{t}}\right.$, $\mathrm{AUC}_{0-24}, \mathrm{AUC}_{0-\infty}$ ) for Test ( $\mathrm{A}$ and $\mathrm{B}$ ) and Reference $(\mathrm{R})$ formulations were analysed using a mixed effects ANOVA (Analysis of variance) model. Each analysis of variance included calculation of least-squares means (LSM), the difference between the adjusted formulation means and the standard error associated with the difference. The above analyses were done using the appropriate SAS ${ }^{\circledR}$ procedure. The ratio of the test (A or B) and reference (R) product averages (least square means) was calculated for olopatadine by first calculating the differences in the averages (arithmetic means) of the log-transformed data and then taking the antilog of the obtained difference. The comparison of interest was A vs $\mathrm{R}$ and $\mathrm{B}$ vs $\mathrm{R}$, so the ratios was of the form $A / R$ and $B / R$. Ratio of means was expressed as a percentage of the LSM for the reference formulations.

\section{RESULTS}

Fifteen [15] healthy, adult, human male subjects, who met the inclusion and exclusion criteria as described in the protocol, were enrolled in the study. Thirteen [16] subjects completed all the three periods of the study as two subjects were withdrawn from the study (one was withdrawn due to adverse event and another subject failed to comply with requirement of protocol). The mean age of the subjects was $26.07 \pm 6.62 \mathrm{y}$ (ranged from 18-38 y) and mean weight was $57.17 \pm 6.68 \mathrm{Kg}$ (ranged from $46.9-69.5 \mathrm{~kg}$ ). The mean height of the subjects was $167.39 \pm 7.63 \mathrm{~cm}$ (ranged from 156-185 cm).
Demographic data of the subjects are provided in table 1. Data from all the subjects who completed the study were included in the final PK analysis. Vital signs of oral temperature, sitting blood pressure and radial pulse were found to be normal for all the subjects during the course of the study in all the three periods of the study. The clinical examination of all subjects was found to be normal. The study treatments were well tolerated by the study subjects, except one subject who experienced vomiting and epigastric pain and subsequently withdrawn from the study.

Two peaks were observed in the mean plasma concentration and time curve of IR formulation $\mathrm{R}$, this was due to the 12-hourly administration of the formulation. Graphs are presented in fig. 1 and 2. Pharmacokinetic data obtained in the study showed that that $\mathrm{C}_{\max }$ attained by ER test formulations $A$ and $B$ was higher than the $\mathrm{C}_{\max }$ of IR reference formulation $R$. There was statistically significant difference $(\mathrm{p}<0.0001)$ between the $\mathrm{C}_{\max }$ of test formulations $\mathrm{A}$ and $\mathrm{B}$ and reference formulations $R$. In relation to area under the curve, the results demonstrated that the ER formulations showed a similar extent of abortion as compared to the reference formulation and there was no statistically significant difference $(\mathrm{p}<0.0001)$ in AUC of test formulations $\mathrm{A}$ and $\mathrm{B}$ and reference $\mathrm{R}$. All the values for $90 \%$ confidence intervals for log transformed data of $\mathrm{C}_{\max }, \mathrm{AUC}_{0-36}, \mathrm{AUC}_{0-24}$ and $\mathrm{AUC}_{0-\infty}$ were within the stated regulatory bioequivalence range of $80-125 \%[17,19]$ except for $C_{\max }$, therefore, bioequivalence between test products A, B and reference product $\mathrm{R}$ cannot be established. Detailed data are presented in tables 2, 3 and 4.

Table 1: Demographic details of subjects

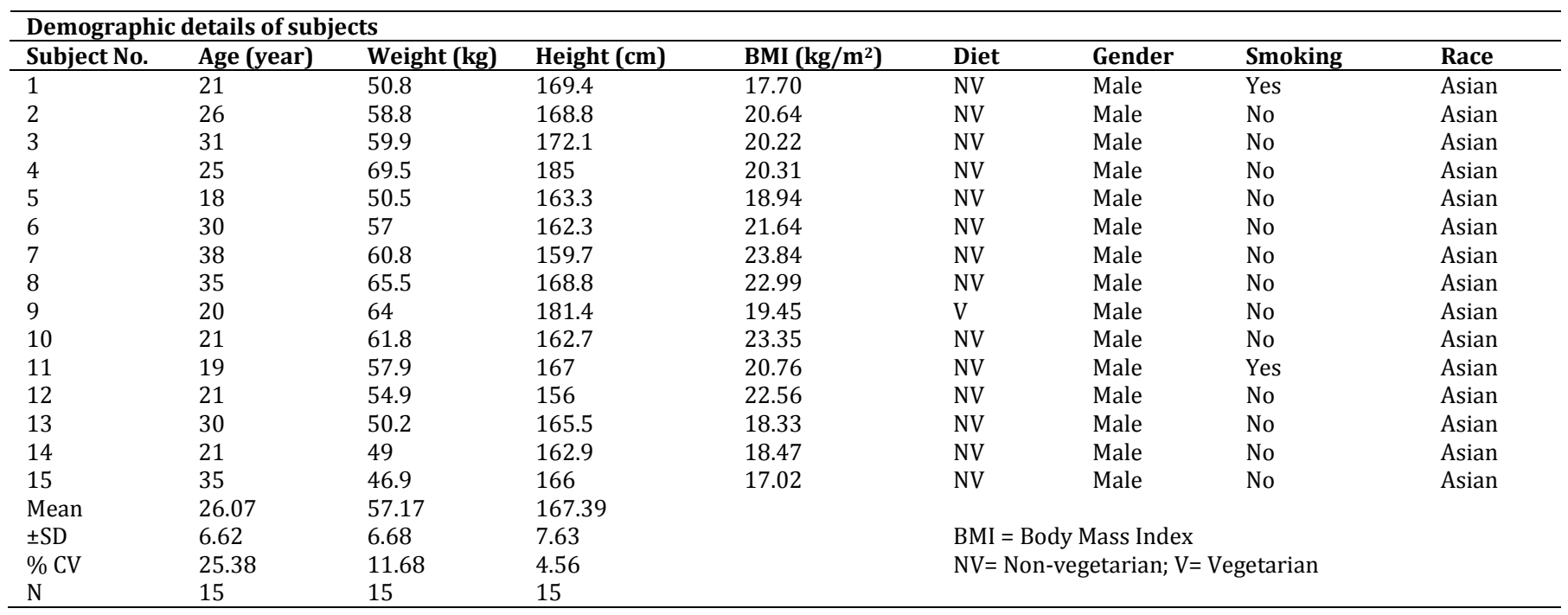

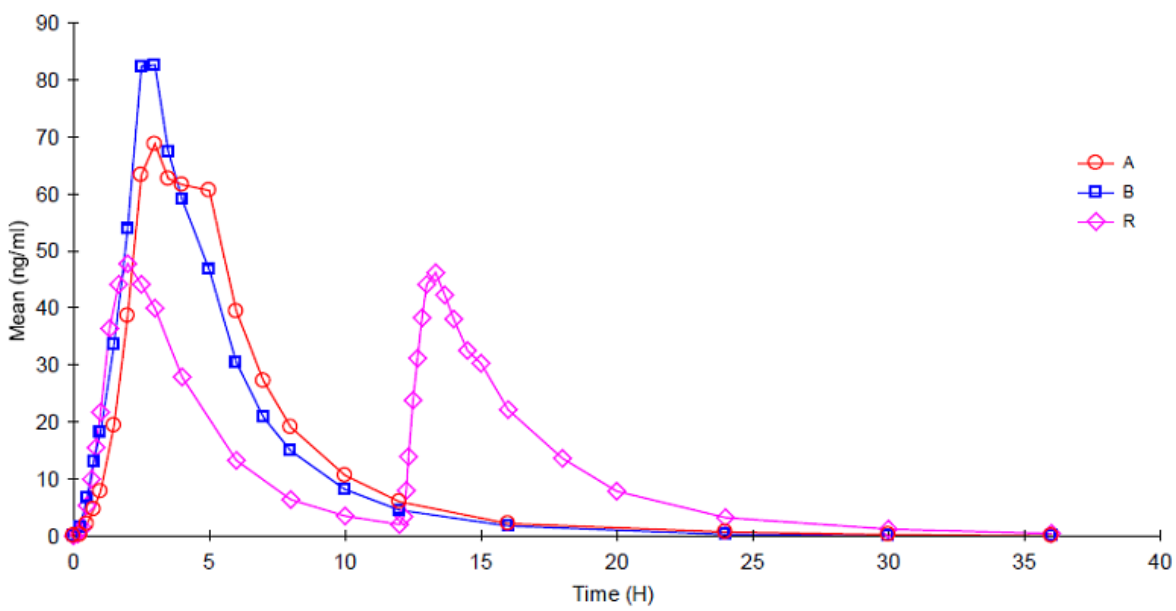

Fig. 1: Linear plot of mean plasma olopatadine concentration $(\mathrm{ng} / \mathrm{ml})$ versus time (h) 


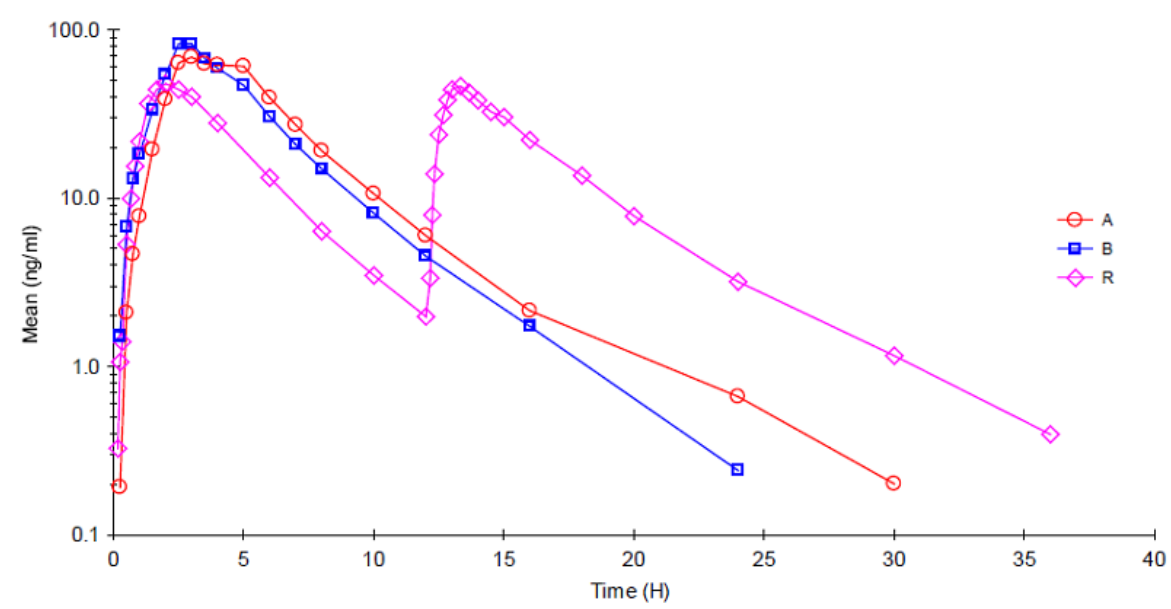

Fig. 2: Semilog plot of mean plasma olopatadine concentration $(\mathrm{ng} / \mathrm{ml})$ versus time $(\mathrm{h})$

Table 2: Pharmacokinetic results $(\mathrm{N}=14)$

\begin{tabular}{lllll}
\hline Product & $\mathbf{C}_{\max }(\mathbf{n g} / \mathbf{m l})^{*}$ & $\mathbf{T}_{\max }(\mathbf{h})^{*}$ & AUC $\left.\mathbf{0 - 2 4}^{*} \mathbf{n g} \cdot \mathbf{h} / \mathbf{m l}\right)^{*}$ & ${\text { AUC } \mathbf{0}-\mathbf{3 6}(\mathbf{n g} . \mathbf{h} / \mathbf{m l})^{*}}^{*}$ \\
\hline $\mathrm{A}$ & $100.33( \pm 29.60)$ & $3.73( \pm 1.13)$ & $387.60( \pm 59.02)$ & $385.56( \pm 62.25)$ \\
$\mathrm{B}$ & $112.38( \pm 37.05)$ & $3.00( \pm 1.00)$ & $377.60( \pm 74.75)$ & $372.81( \pm 75.90)$ \\
$\mathrm{R}$ & $65.18( \pm 17.86)$ & $8.41( \pm 5.78)$ & $403.64( \pm 93.26)$ & $418.27( \pm 100.51)$ \\
\hline
\end{tabular}

*Mean values are presented

Table 3: Summary statistics of different $P K$ parameters $(\mathrm{N}=14)$

\begin{tabular}{|c|c|c|c|}
\hline Parameters & Product A & Product B & Product $\mathrm{R}$ \\
\hline Mean $\mathrm{C}_{\max }(\mathrm{ng} / \mathrm{ml})^{*}$ & 95.6178 & 106.6336 & 62.9773 \\
\hline Mean $\mathrm{AUC}_{0-36}$ (ng. h/ml)* & 380.80621 & 365.64849 & 407.55943 \\
\hline Mean AUC $0-24$ (ng. h/ml)* & 383.36735 & 370.84072 & 394.36214 \\
\hline Mean $\mathrm{UUC}_{0-\infty}$ (ng. h/ml)* & 388.34376 & 372.16152 & 414.87231 \\
\hline
\end{tabular}

*Log-transformed parameters, the antilog of the mean (i.e. the geometric mean) is reported

Table 4: $90 \%$ confidence intervals for $\log$ transformed data of test $v / s$ reference

\begin{tabular}{lllll}
\hline Formulations & \multicolumn{1}{l}{ Parameters } & & \\
\cline { 2 - 5 } & $\mathbf{C}_{\max }$ & AUC $\mathbf{0} \mathbf{3}$ & AUC $_{\mathbf{0}-\mathbf{2} 4}$ & AUC $_{\mathbf{0}-\infty}$ \\
\hline A v/s R & $127.41-179.17$ & $86.37-96.05$ & $90.13-99.93$ & $86.75-96.12$ \\
B v/s R & $142.25-198.30$ & $85.11-94.39$ & $89.34-98.79$ & $85.26-94.22$ \\
\hline
\end{tabular}

\section{DISCUSSION}

Olopatadine is available in its conventional IR form to be administered twice a day. An undoubted advantage of ER formulations over conventional dosage forms is improved patient compliance; compliance improves dramatically as prescribed dose frequency decreases [3-6]. Apart from improved patient compliance, there is decreased fluctuation in steady state levels leading to better control of disease condition and reduced intensity of local and systemic side effects $[5,16,17]$.

In this study, higher $\mathrm{C}_{\max }$ values of test formulations $\mathrm{A}$ and $\mathrm{B}$ were reported as compared to reference formulation $\mathrm{R}$, there was statistically significant difference in $\mathrm{C}_{\max }$ between reference formulation and test formulation $\mathrm{A}$ and $\mathrm{B}$ as $\mathrm{A} / \mathrm{R}$ and $\mathrm{B} / \mathrm{R}$ ratio values were 151.09 and 167.96 respectively which were higher than the defined regulatory range of $80 \%$ to $125 \%$. In a comparative bioavailability study conducted to examine the pharmacokinetics of prochlorperazine immediate release tablet and sustained release tablet in healthy, adult, male volunteers, the reported $\mathrm{C}_{\max }$ values for sustained release tablet and immediate release tablet were 297.89 and $218.41 \mathrm{ng} / \mathrm{ml}$ respectively [18], the similar pattern is shown in our study where the Cmax values of the sustained release formulations were higher than the immediate release.
The reason for higher Cmax of test formulations $A$ and $B$ than the reference formulation was possibly due to the fact that in first 2-3 $\mathrm{h}$ these formulations behaved similar to IR formulation. Another possible reason of the higher Cmax values of extended release test formulations could be dose dumping. Dose dumping is defined as unintended, rapid drug release in a short period of time of the entire amount or a significant fraction of the drug contained in a modified release dosage from. Dose dumping can pose a significant risk to patients, either due to safety issue or diminished efficacy or both. Generally, dose dumping is observed due to a compromise of the release-rate-controlling mechanism [19]. It is often reported when a modified oral dosage from is conjunction with high fat food or alcohol. Hendeles et al. reported dose dumping phenomenon in a study conducted on theophylline extended release tablets taken under fed conditions. They reported that food caused precipitous doe-dumping resulting in dose normalized peak levels in in the serum that averaged 2.3 times higher than after a fasting dose [20]. Another study of nifedipine ER tablets in healthy volunteers reported an increase in plasma concentration of the test drug after a high fat breakfast. The test product developed a dose-dumping effect after the intake of food. This phenomenon went along with a loss in modified release characteristics [21].

In our study, extended release formulations showed a similar pattern which indicates a possible dose dumping effect. Major 
portion of olopatadine from both the extended release test formulations was released in first few hours leading to a failure in the modified release characteristics of the formulation. However, these higher Cmax values of extended release formulations cannot be confidently attributed to the dose dumping phenomenon, as to confirm this we need to perform a similarly designed study in fasted state with a similar set of subjects. Another noteworthy observation in the study was that the maximum Cmax values for both the test formulations were reported from the same subject who is a regular smoker, so it is possible that regular smoking may have produced some changes in the normal physiological processes of the subject which ultimately caused the higher release of the of the drug from the ER formulations. Numerous drug interactions have been identified with tobacco smoke [22] and many regulatory agencies like Canadian, European and WHO recommend that preferably a non-smoker should be included in the bioequivalence studies [23$25]$. But this can only be confirmed when another study in planned with both smoker and non-smoker subjects to study the effect of smoking on the pharmacokinetic parameters of olopatadine.

Nonetheless, these higher Cmax values of test formulation didn't pose any safety issue, as only one subject in test arm reported postdose gastrointestinal adverse events which were not serious in nature and subject recovered without sequelae.

\section{CONCLUSION}

Based on pharmacokinetic and clinical results, it can be summarized that extended release formulation A and B achieved similar AUC as compared to reference drug but in both the test formulations most of the drug got released in initial few hours resulting in higher Cmax values but there were no safety concerns. Although the extended release formulations have showed a similar extent of absorption, but the products need to be reformulated in such a manner that slow drug release can be achieved as shown by lower Cmax and longer Tmax. In conclusion, we can say that though the study drugs did not meet the bioequivalence criteria set by regulatory agencies, but this study gave an insight about PK properties of olopatadine extended release formulation and given an idea about effect of smoking on the PK profile of olopatadine which can be studied in future.

\section{ACKNOWLEDGMENT}

We would like to acknowledge Ranbaxy Research Laboratories limited, Gurgaon, India for the study samples and for allowing to use their clinical and bioanalytical facility.

\section{FUNDING}

No funding was received for the study.

\section{AUTHORS CONTRIBUTIONS}

All the authors have contributed equally.

\section{CONFLICT OF INTERESTS}

All authors declare that they have no conflict of interest.

\section{REFERENCES}

1. Hoffman A. Pharmacodynamic aspects of sustained release preparations. Ad Drug Delivery Rev 1998;33:185-99.

2. Talan DA, Naber KG, Palou J, Elkharrat D. Extended-release ciprofloxacin (Cipro XR) for treatment of urinary tract infections. Int J Antimicrob Agents 2004;23:54-66.

3. Pullar T, Birtwell AJ, Wiles PG, Hay A, Feely MP. Use of a pharmacologic indicator to compare compliance with tablets prescribed to be taken once, twice, or three times daily. Clin Pharmacol Ther 1998;44:540-5.

4. Eisen SA, Miller DK, Woodward RS, Spitznagel E, Przybeck TR. The effect of prescribed daily dose frequency on patient medication compliance. Arch Intern Med 1990;150:1881-4.

5. Brun J. Patient compliance with once-daily and twice-daily oral formulations of 5 isosorbide mononitrate: a comparative study. J Int Med Res 1994;22:266-72.
6. Claxton AJ, Cramer J, Pierce C. A systematic review of the associations between dose regimens and medication compliance. Clin Ther 2001;23:1296-310.

7. Shargel L, Wu Pong S, Yu A. Applied biopharmaceutics and pharmacokinetics. (6th Ed.). The McGraw Hill Companies Inc. USA; 2010.

8. Ohmori K, Hayashi K, Kaise T, Ohshima E, Kobayashi S, Yamazaki $\mathrm{T}$, et al. Pharmacological, pharmacokinetic and clinical properties of olopatadine hydrochloride, a new antiallergic drug. Japan J Pharmacol 2000;88:379-97.

9. Kaliner MA, Oppernheimer J, Farrar JR. Comprehensive review of olopatadine: the molecule and its clinical entities. Allergy Asthma Proc 2010;31:112-9.

10. Guidance for industry: Bioavailability and bioequivalence studies for orally administered drug products--general considerations. U. S. department of health and human services, food and drug administration (FDA), centre for drug evaluation and research (CDER); 2003.

11. Guidelines for bioavailability and bioequivalence studies. Central drugs standard control organization (CDSCO), Ministry of health and family welfare, Government of India, New Delhi; 2005.

12. USFDA Code of Federal Regulation. 21 CFR 320. Vol. 5; 2012. Available from: http://www.accessdata.fda.gov/scripts/cdrh/cfdocs/cfcfr/CFR Search.cfm?CFRPart $=320$ andshowFR $=1$ [Last accessed on 10 Aug 2020]

13. Ethical guidelines for biomedical research on human participants. Indian council for medical research (ICMR), Government of India, New Delhi; 2006.

14. Declaration of Helsinki. 59th World medical association (WMA) general assembly, Seoul; 2008.

15. Guidance for industry: Bioanalytical method validation. US department of health and human services, food and drug administration (FDA), center for drug evaluation and research (CDER); 2001.

16. Beaulieu AD, Peloso PM, Haraoui B, Bensen W, Thomson G, Wade J, et al. Once-daily, controlled-release tramadol and sustained-release diclofenac relieve chronic pain due to osteoarthritis: a randomized controlled trial. Pain Res Manag 2008;3:103-10.

17. Duchier J, Iannascoli F, Safar M. Antihypertensive effect of sustained-release isosorbide dinitrate for isolated systolic systemic hypertension in the elderly. Am J Cardiol 1987;60:99102.

18. Venkatesh DN, Sankar S, Meyyanathan SN, Muralidharan S, Shanmugam R, Elango $\mathrm{K}$, et al. Bioavailability studies on developed prochloperazine maleate sustained release tablets by HPLC. J Bioanal Biomed 2009;1:54-7.

19. Meyer RJ, Hussain AS. Awareness topic: mitigating the risk of ethanol induced dose dumping from oral sustained/controlled dosage forms, FDA's ACPS meeting; 2005.

20. Hendeles L, Weinberger M, Milavetz G, Hill M 3rd, Vaughan L. Food-induced dose-dumping from a once-a-day theophylline product as a cause of theophylline toxicity. Chest 1995;57:75865.

21. Wonnemann M, Schug B, Anschütz M, Brendel E, De Nucci G, Blume H. Comparison of two marketed nifedipine modified release formulations: An exploratory clinical food interaction study. Clin Ther 2008;30:48-58.

22. Kroon LA. Drug interactions with smoking. Am J Health Syst Pharm 2007;64:1917-21.

23. Guideline on the investigation of bioequivalence. Committee for medicinal products for human use (CHMP), European medicines agency (EMA), London; 2010.

24. Guidance document: Conduct and analysis of comparative bioavailability studies. Therapeutic products directorate (TPD), Health Canada; 2012.

25. World Health Organization (WHO) Technical report series, no. 937. WHO expert committee on specifications for pharmaceutical preparations, WHO, Geneva, Switzerland; 2006. 